\title{
EXPERIENCE WITH SUPERCONDUCTING CAVITY OPERATION IN THE TESLA TEST FACILITY
}

\author{
M. Pekeler for the TESLA Collaboration* \\ Deutsches Elektronen-Synchrotron DESY, D-22603 Hamburg, Germany
}

\begin{abstract}
A description of the TESLA Test Facility, which has been set up at DESY by the TESLA Collaboration, will be given. Measurements of the superconducting 9-cell cavities in vertical and horizontal test cryostats will be presented, as well as the experience with the first two accelerator modules in the TTF linac. Future cavity R\&D efforts will be described.
\end{abstract}

\section{INTRODUCTION}

A linear $\mathrm{e}^{+} \mathrm{e}^{-}$collider with a center-of-mass energy of $\geq 500 \mathrm{GeV}$ would be an ideal machine to search for further fundamental constituents of matter and their interactions and to address the problem of mass generation in the Standard Model. Among the different designs (NLC, JLC, VLEPP, CLIC \& TESLA), TESLA is the only one using superconducting cavities and a low radio frequency of $1.3 \mathrm{GHz}$. The high conversion efficiency from primary to beam power and the small emittance dilution makes the superconducting version an ideal choice for high luminosity operation [1]. The TESLA $500 \mathrm{GeV}$ collider design is based on nine-cell cavities with a gradient of $25 \mathrm{MV} / \mathrm{m}$ at a quality factor $Q$ of more than $5 \cdot 10^{9}$ [2]. An important feature is the integrated $\mathrm{X}$ ray Free Electron Laser (FEL) working on the Self-Amplified Spontaneous Emission (SASE) principle. This FEL will produce a photon beam at Angstrom wavelengths with peak brilliance exceeding that of third generation synchrotron radiation sources by 10 orders of magnitude.

\section{THE TESLA TEST FACILITY}

In 1992 the TESLA Collaboration decided to set up the TESLA Test Facility (TTF) [3] at DESY comprising the complete infrastructure for the treatment, assembly and test of 9-cell superconducting cavities and a superconducting linac for a fully integrated systems test with beam. The

* TESLA Collaboration: Armenia: Yerevan Pysics Institute, P.R. China: IHEP Beijing, Tsinghua Univ. Beijing, Finland: Inst. of Physics Helsinki, France: CEA/DSM Saclay, IN2P3 Orsay, Germany: MaxBorn-Inst. Berlin, DESY Hamburg and Zeuthen, Univ. Wuppertal, Univ. Hamburg, Univ. Frankfurt, GKSS Geesthacht, FZ Karlsruhe, TU Darmstadt, TU Berlin, TU Dresden, RWTH Aachen, Univ. Rostock, Italy: INFN Frascati, INFN Legnaro, INFN Milano, INFN Roma, Univ. Roma II, Poland: Polish Acad. of Sciences, Univ. Warsaw, INP Cracow, Univ. of Mining \& Metallurgy, Polish Atomic Energy Agency, Soltan Inst. for Nuclear Studies, Russia: JINR Dubna, IHEP Protvino, INP Novosibirsk, INR Troitsk, USA: Argonne National Lab., Cornell Univ., FNAL, UCLA aim was to achieve gradients of $15 \mathrm{MV} / \mathrm{m}$ in a first step and to gradually approach the $25 \mathrm{MV} / \mathrm{m}$ design gradient of the linear collider.

\subsection{The TTF linac}

The original proposal was to built a $500 \mathrm{MeV}$ superconducting linac as a test bed for the cavities and RF systems foreseen for TESLA, but during the last years the design of the TTF Linac was extended to include from the beginning the important option of a Free Electron Laser in the Vacuum Ultraviolet regime [4]. Two injectors have been in use, a $250 \mathrm{keV}$ thermionic gun [5] producing an average current of $8 \mathrm{~mA}$ at low bunch charge, and a $4 \mathrm{MeV}$ laser-driven RF photoinjector [6] with the same average current, but a high bunch charge of $8 \mathrm{nC}$ corresponding to the TESLA specifications. The electrons are captured by a superconducting nine-cell cavity (see figure 1) providing an energy gain of $13 \mathrm{MeV}$. In 1997 the first stage of the linac was successfully commissioned with the thermionic injector and one cryomodule containing eight 9-cell cavities with an average gradient of $15 \mathrm{MV} / \mathrm{m}$ [7]. The second cryomodule was installed in summer 1998. All cavities exceeded a gradient of $20 \mathrm{MV} / \mathrm{m}$ in the vertical test, four reached even the TESLA goal of $25 \mathrm{MV} / \mathrm{m}$. The cavities of the third module which is presently being assembled will all operate at $25 \mathrm{MV} / \mathrm{m}$.

Together with the installation of module 2, the thermionic gun was replaced by the photoinjector and a bunch compressor was installed between the two modules. The undulator magnet together with module 3 will be mounted this summer. The proof-of-principle experiment for the SASE type FEL is planned for the fall. In Phase II the TTF linac will be equipped with 5 more cryomodules, a second bunch compressor and a three times longer undulator. The completion of a VUV FEL user facility is planned for the fall of 2001 .

\subsection{The TTF infrastructure}

The TTF infrastructure for cavity preparation and test [8] was completed in 1995 and is composed of a complex of clean rooms (from class 10000 to class 10), a chemical etching facility and an ultra-clean water supply. A UHV furnace is available to improve the thermal conductivity of the cavity via heat-treatment at $1400^{\circ} \mathrm{C}$ in the presence of titanium gettering. The last step of cavity preparation is a high pressure (100 bar) rinsing with ultra pure water. 

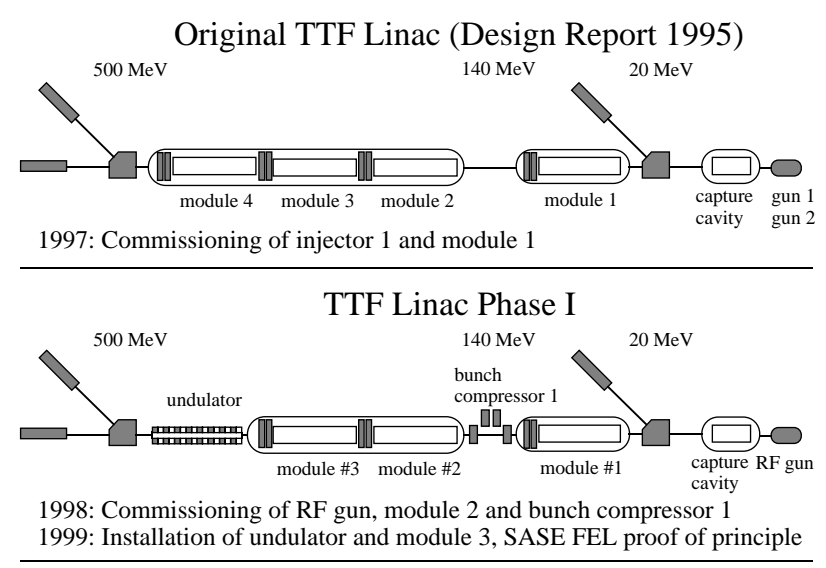

TTF Linac Phase II

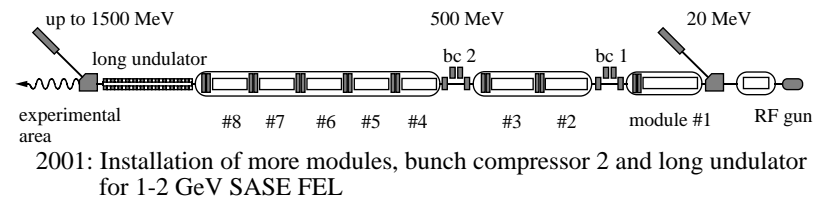

Figure 1: Development stages of the TTF linac design.

All cavities are tested in superfluid helium of $2 \mathrm{~K}$ in a vertical bath cryostat. The possibility exists to apply high peak power processing [9] as well as temperature mapping of the outer cavity surface [10]. Cavities having passed the vertical test are welded into their helium tank. The fully assembled cavity can be tested in a horizontal cryostat in pulsed power mode ( $500 \mu$ s rise time, $800 \mu$ s flattop time at a $10 \mathrm{~Hz}$ repetition rate). The performance of the main power coupler, the higher-order-mode couplers and the cold tuning mechanism is checked here before the cavity is installed into the cryomodule.

The cavities can be tested also after installation in the linac. As in the horizontal cryostat, the quality factor $Q$ is determined by measuring the dynamic heat-load. The cryoplant of the TTF permits a heat-load measurement at $2 \mathrm{~K}$ with a resolution of $0.05 \mathrm{~W}$ (as a reference, a cavity operated at $25 \mathrm{MV} / \mathrm{m}$ and $Q=5 \cdot 10^{9}$ gives a heat dissipation of $1.8 \mathrm{~W}$ with the TTF pulsed mode).

\section{CAVITY PREPARATION AND TESTS}

A cross section of the TTF nine-cell cavity is shown in figure 2. The cavities are fabricated from RRR 300 niobium by electron-beam welding of half cells that are deep-drawn from niobium sheet metal. Up to now 55 cavities have been ordered at 4 European companies. The first series of 28 cavities were ordered in 1994, the second series of 27 in 1997. So far, 41 cavities have been tested in the vertical and 18 cavities in the horizontal cryostat.

The presently used cavity preparation at DESY consists of the following steps:

- Removal of a damage layer from the inner cavity surface by $80 \mu \mathrm{m}$ Buffered Chemical Polishing (BCP)

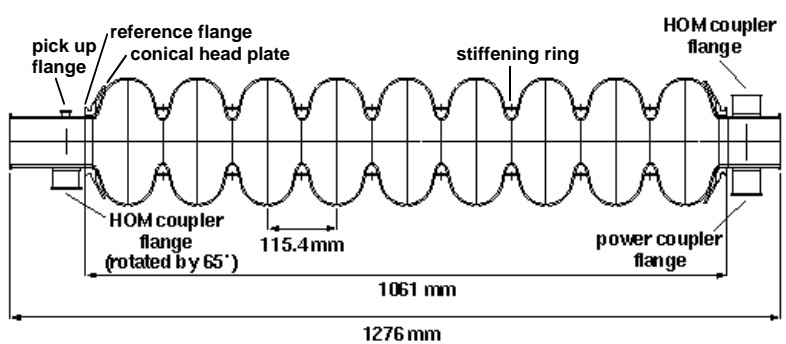

\begin{tabular}{|l|c|}
\hline Effective length & $1036 \mathrm{~mm}$ \\
\hline Aperture diameter & $70 \mathrm{~mm}$ \\
\hline Coupling cell to cell & $1.98 \%$ \\
\hline$E_{\text {peak }} / E_{\text {acc }}$ & 2.0 \\
\hline$B_{\text {peak }} / E_{\text {acc }}$ & $4.2 \mathrm{mT} /(\mathrm{MV} / \mathrm{m})$ \\
\hline R/Q per cavity & $1036 \Omega$ \\
\hline$\Delta f / \Delta L$ & $315 \mathrm{kHz} / \mathrm{mm}$ \\
\hline Cavity bandwidth $\left(Q_{\text {ext }}=3 \cdot 10^{6}\right)$ & $433 \mathrm{~Hz}$ \\
\hline
\end{tabular}

Figure 2: Cross section and some design parameters of the $1.3 \mathrm{GHz}$ TTF 9-cell cavity

using a mixture of $\mathrm{HF}(48 \%), \mathrm{HNO}_{3}(65 \%)$ and $\mathrm{H}_{3} \mathrm{PO}_{4}(85 \%)$ in the ratio $1: 1: 2$. This is followed by rinsing with ultrapure water until the resistivity of the water is higher than $18 \mathrm{M} \Omega \mathrm{cm}$.

- Removal of $30 \mu \mathrm{m}$ from the outer surface by BCP.

- A 2 hours heat-treatment at $800{ }^{\circ} \mathrm{C}$ for hydrogen degasing and recrystallisation.

- A 4 hours heat-treatment at $1400{ }^{\circ} \mathrm{C}$ with titanium getter for improvement of the thermal conductivity and homogenization of the niobium.

- Removal of the titanium layer by $80 \mu \mathrm{m}$ inner and $30 \mu \mathrm{m}$ outer BCP.

- Tuning to correct frequency and field flatness.

- Final $20 \mu \mathrm{m}$ removal from the inner surface by BCP.

- High pressure rinsing with 100 bar ultrapure water.

- Drying by laminar flow in class 10 cleanroom, assembly of all flanges, leak-check.

- 2 times high pressure water rinsing, drying by laminar flow and assembly of input antenna.

\subsection{Vertical test results}

The vertical test results are listed in table 1 . Several cavities of the first series reached gradients up to $29 \mathrm{MV} / \mathrm{m}$, but the distribution of achieved gradients is very wide. In cavities with low performance defects in the welds or in the bulk niobium were found [11].

To avoid such defects, the welding technique was improved and all niobium sheets used for the second series were eddy current scanned [12] to eliminate foreign material inclusions. Almost all cavities of the second production reached gradients above $20 \mathrm{MV} / \mathrm{m}$ (see table 1). Two cavities (S34 and Z49) suffered from field emission and will be retested soon after a new high pressure water rinse. Cavity $\mathrm{C} 43$ shows a quench at a repaired equator weld where a hole was blown during electron beam welding. The ma- 
Table 1: Performance of TTF cavities in vertical tests. ${ }^{+}$: limited by available cw RF power (no quench observed).

\begin{tabular}{|c|c|c|c|}
\hline cavity & $\begin{array}{c}E_{\mathrm{acc}} \\
\mathrm{MV} / \mathrm{m}\end{array}$ & $\begin{array}{r}Q_{0} \\
10^{9}\end{array}$ & status / comment \\
\hline \multicolumn{4}{|c|}{ first production } \\
\hline P1 & $29.1^{+}$ & 6 & prototype cavity \\
\hline $\mathrm{P} 2$ & 16.3 & 22 & prototype cavity \\
\hline D1 & 24.7 & 17 & linac operation (module 1 ) \\
\hline D2 & 21.9 & 4 & module 1 \\
\hline D3 & 25.6 & 29 & module 1 \\
\hline D4 & 13.5 & 16 & module 1 \\
\hline D5 & 8.6 & 24 & material defect \\
\hline D6 & 13.6 & 12 & material defect \\
\hline S7 & 13.8 & 8 & module 1/ weld defects \\
\hline S8 & 12.5 & 12 & module 1 / weld defects \\
\hline S9 & 11.4 & 11 & weld defects \\
\hline S10 & 14.2 & 16 & module 1 / weld defects \\
\hline S11 & 13.5 & 13 & module 1 / weld defects \\
\hline $\mathrm{S} 12$ & 12.6 & 13 & used at FNAL / weld defects \\
\hline A14 & 6.4 & 11 & quench at repaired weld \\
\hline A15 & $23.0^{+}$ & 4 & module 2 \\
\hline A16 & 20.8 & 6 & field emission \\
\hline C19 & 22.1 & 2 & linac (capture cavity) \\
\hline $\mathrm{C} 21$ & $29.3^{+}$ & 8 & module 2 \\
\hline $\mathrm{C} 22$ & 20.2 & 21 & module 2 / weld defect \\
\hline $\mathrm{C} 23$ & $25.3^{+}$ & 8 & module 2 \\
\hline $\mathrm{C} 24$ & $19.7^{+}$ & 5 & module 2 \\
\hline $\mathrm{C} 25$ & $28.4^{+}$ & 9 & module 2 \\
\hline $\mathrm{C} 26$ & $21.4^{+}$ & 4 & module 2 \\
\hline $\mathrm{C} 27$ & $26.7^{+}$ & 8 & module 2 \\
\hline \multicolumn{4}{|c|}{ second production } \\
\hline S28 & $25.3^{+}$ & 6 & module 3 \\
\hline S29 & $26.7^{+}$ & 6 & module 3 \\
\hline $\mathrm{S} 30$ & $28.4^{+}$ & 7 & module 3 \\
\hline S31 & 28.1 & 4 & \\
\hline $\mathrm{S} 32$ & $26.5^{+}$ & 7 & module 3 \\
\hline S33 & 23.9 & 7 & \\
\hline S34 & $14.4^{+}$ & 2 & field emission \\
\hline D37 & 20.3 & 5 & \\
\hline D38 & 19.5 & 3 & \\
\hline D39 & $25.2^{+}$ & 7 & module 3 \\
\hline D40 & $22.8^{+}$ & 5 & module 3 \\
\hline D41 & $23.3^{+}$ & 5 & module 3 \\
\hline D42 & 24.6 & 7 & module 3 \\
\hline $\mathrm{C} 43$ & 12.9 & 20 & quench at repaired weld \\
\hline $\mathrm{C} 44$ & $25.5^{+}$ & 6 & \\
\hline Z49 & $18.0^{+}$ & 2 & field emission \\
\hline
\end{tabular}

jority of the cavities reached their excellent performance already in the first vertical test. It is only occassionally necessary to repeat preparation steps.

The average gradient of all cavities is $20.5 \mathrm{MV} / \mathrm{m}$. Neglecting cavities with identified material or fabrication errors gives an average gradient of $23.1 \mathrm{MV} / \mathrm{m}$.
Figure 3 shows the time development of the best vertical test results. Since 1997 fabrication and material defects have been almost eliminated. Practically all of the new cavities reach gradients between 20 and $30 \mathrm{MV} / \mathrm{m}$ with an average of $25 \mathrm{MV} / \mathrm{m}$.

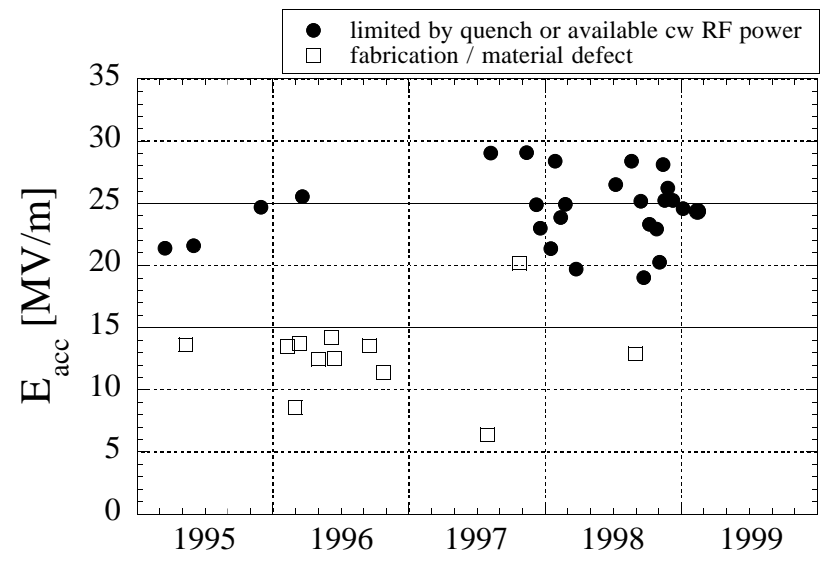

Figure 3: Time development of maximum gradient achieved in TTF 9-cell cavities.

\subsection{Horizontal tests}

After the successful vertical test, the helium vessel is welded to the conical head plates of the cavity (see figure 2). A $20 \mu \mathrm{m}$ removal from the inner surface and a careful high pressure rinse follows. The last preparation step is the delicate assembly of the main power coupler.

The average gradient obtained in the 18 cavities tested in the horizontal cryostat was $22.5 \mathrm{MV} / \mathrm{m}$ and does not differ from the average value these cavities reached during the vertical tests $(22.3 \mathrm{MV} / \mathrm{m})$. The best horizontal test result was $33 \mathrm{MV} / \mathrm{m}$ with $Q=4 \cdot 10^{9}$. Most of the good cavities are limited by RF breakdown in the main power coupler. Four of the eight cavities selected for module 3 were tested in the horizontal cryostat and all exceeded $25 \mathrm{MV} / \mathrm{m}$ with quality factors above $5 \cdot 10^{9}$.

\section{OPERATION OF CAVITIES IN THE TTF LINAC}

The two modules installed in the TTF linac are supplied with RF power by one $5 \mathrm{MW}$ klystron. In the future it is foreseen to supply 32 cavities by one $10 \mathrm{MW}$ klystron (as in the TESLA design). The cavities are not controlled individually but only the sum of the electric field vectors of all cavities is regulated by a digital RF control system. In addition to the feedback control which suppresses stochastic errors, an adaptive feedforward is applied to correct for repetitive perturbations, induced by beam loading and dynamic Lorentz force detuning [13]. The remaining errors from noise and other sources are small and require only a low gain in the feedback loop. An amplitude stability of 

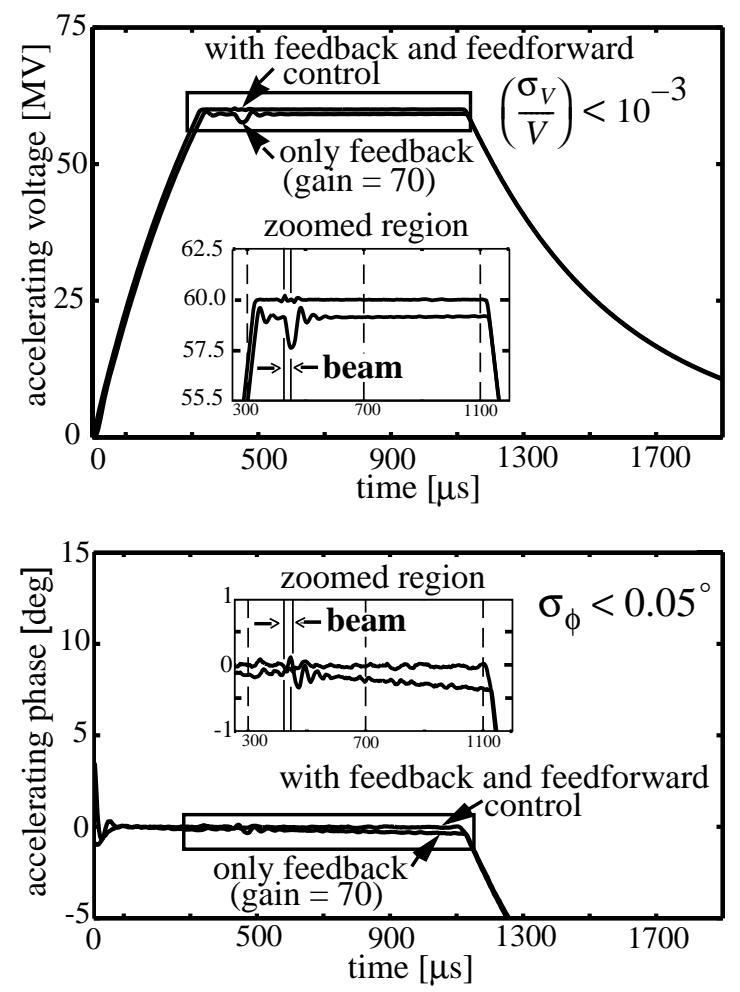

Figure 4: RF control system performance without and with adaptive feedforward. The beam pulse had a length of $30 \mu \mathrm{s}$.

$<10^{-3}$ and a phase stability of $<0.05^{\circ}$ was achieved for the vector sum (see figure 4).

The cavities installed in module 1 reached an average gradient of $15 \mathrm{MV} / \mathrm{m}$, two of them were already close to or above the TESLA specs $\left(25 \mathrm{MV} / \mathrm{m}\right.$ with $\left.Q>5 \cdot 10^{9}\right)$ [7].

After the installation of module 2, there was only limited time for main coupler conditioning. No measurements of individual cavities were possible but an integral check of the whole module, with all cavities operating at the same gradient. With the full TTF pulse $(10 \mathrm{~Hz}, 500 / 800 \mu \mathrm{s}$ rise/flat-top time) $20 \mathrm{MV} / \mathrm{m}$ could be reached. The gradient could not be increased further because of coupler breakdowns. Heavy field emission was detected in one of the cavities. It was decided to apply in-situ high peak power processing (HPP) prior the second test and fields up to $29 \mathrm{MV} / \mathrm{m}$ were reached in this cavity. After the HPP the heat load of module 2 with all cavities operating at $20 \mathrm{MV} / \mathrm{m}$ was $6.5 \mathrm{~W}$, corresponding to an average quality factor of $Q=6 \cdot 10^{9}$. By detuning the cavity in which still some field emission was present the cryogenic load reduced to $2.9 \mathrm{~W}$ giving at $20 \mathrm{MV} / \mathrm{m}$ a $Q$ of $1.3 \cdot 10^{10}$ of the remaining 7 cavities.

\section{FUTURE CAVITY R\&D}

From the good test results of TTF cavities after mid 1997 one can conclude that the TESLA goal of $25 \mathrm{MV} / \mathrm{m}$ in 9- cell resonators has been established with the present fabrication and preparation methods. However for a possible energy upgrade of TESLA there is a strong motivation to push the cavities closer to the physical limit of $50 \mathrm{MV} / \mathrm{m}$ which is determined by the critical magnetic field of the superconductor niobium.

There are three main effects which prevent us from reaching gradients well beyond $25 \mathrm{MV} / \mathrm{m}$ : thermal breakdown at material defects, field emission and $Q$-drop at high fields.

Occasionally we observe quenches at about $25 \mathrm{MV} / \mathrm{m}$. New vertical tests with temperature mapping of the outer cavity surface are necessary in order to find out, if the delicate equator welds or very small foreign material inclusions are responsible for the thermal breakdown. In addition the eddy current scanning apparatus [12] was improved significantly for a better diagnostic of polluted niobium sheets with tiny defects.

With the present preparation techniques, field emission may be observed at gradients above $20 \mathrm{MV} / \mathrm{m}$. Efforts are undertaken to further reduce field emission by improvement of the high pressure rinsing system and a better in-situ particle control during the assembly in the cleanroom.

A new type of field limitation, first observed in single cell cavities at Saclay, and recently also seen at DESY, is the decrease of the quality factor at fields around $25 \mathrm{MV} / \mathrm{m}$ without any evidence for field emission (see figure 5). In a collaboration between Saclay and KEK it was shown that electropolishing of the surface reduces this $Q$-drop and enhances the high-field capability compared to a buffered chemical polished surface [14]. An R\&D effort has been launched in collaboration with CERN, KEK, Saclay and an industrial company to apply electropolishing to the 9cell TESLA structures.

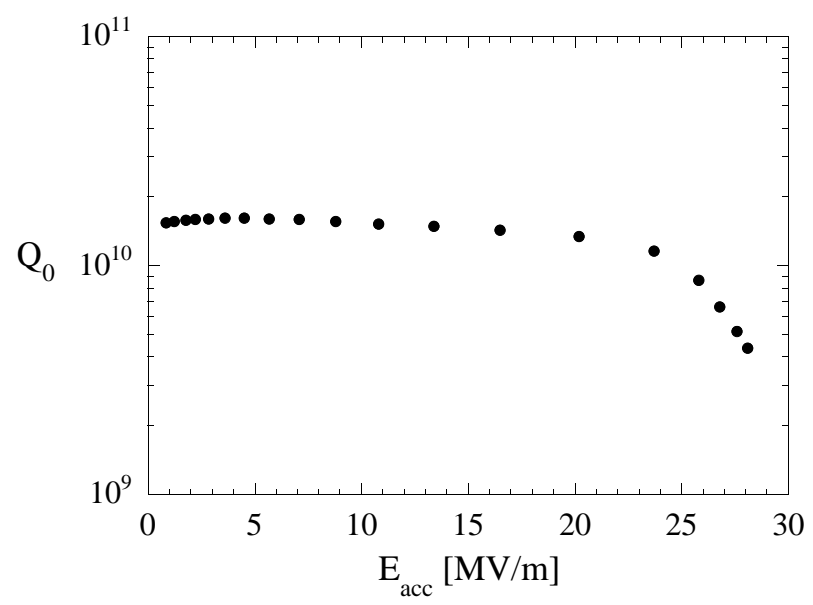

Figure 5: Observation of $Q$-drop at high gradient without any evidence of field emission in a TTF 9-cell cavity.

New cavity fabrication methods [15] like spinning or hydroforming [16] are under development and have the potential of reducing the costs. First test results on prototype cavities are promising (figure 6). A spun five-cell $1.5 \mathrm{GHz}$ 


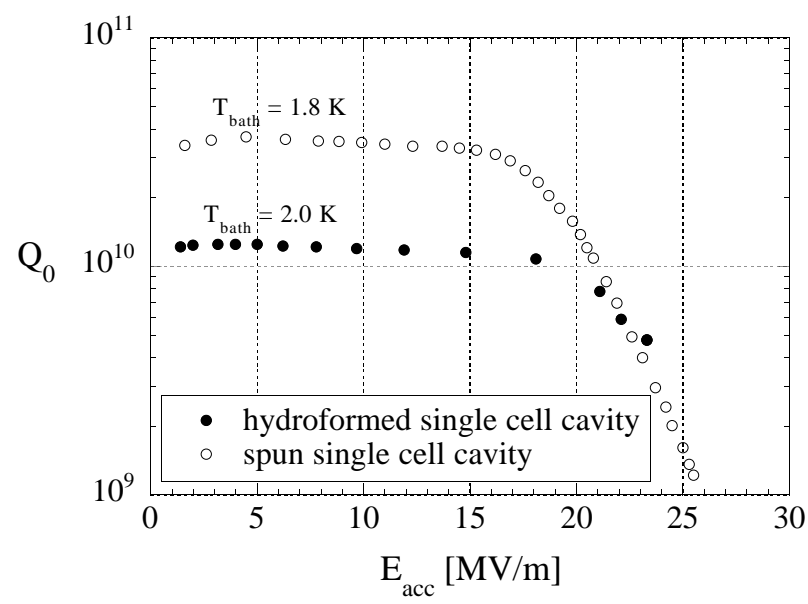

Figure 6: Vertical test results of prototype $1.3 \mathrm{GHz}$ single cell cavities manufactured by hydroforming and a spinning technique.

cavity measured at CEBAF reached even $30 \mathrm{MV} / \mathrm{m}$ [17].

A new way of cavity reinforcement is plasma jet spraying of copper or other metals onto a niobium cavity [18]. The stiffening rings (see figure 2), presently used to counteract the Lorentz forces in pulsed operation, can probably be eliminated and additional stiffening at the equator can be achieved using this method.

In the superstructure concept [19] several multi-cell cavities are coupled to each other by a shorter and larger diameter beam pipe. Only one main input coupler is foreseen for such a group of cavities. This year a design for a $4 \times 7$-cell superstructure will be completed and 7-cell $1.3 \mathrm{GHz}$ superstructure cavities will be ordered. The number of main couplers can be reduced to $1 / 3$ as compared to the present TESLA design and the filling factor, the ratio of the linac active length to the total length, is increased from 0.66 to 0.76 . We hope to test a superstructure with beam in 2001, in order to study beamloading and wakefield effects.

\section{CONCLUSIONS}

The results obtained so far in the framework of TTF are very encouraging and the technical possibility to built TESLA is becoming reality. In particular the cavities are now routinely reaching the TESLA requirements of $25 \mathrm{MV} / \mathrm{m}$ at $Q>5 \cdot 10^{9}$. No degradation of cavity performance is found between vertical test, horizontal test and after installation in the linac.

\section{ACKNOWLEDGEMENTS}

It is a very rewarding experience for me to work in the international TESLA collaboration. I want to thank all my colleagues for stimulating discussions.

Special thanks are given to P. Schmüser for carefully reading the manuscript.

\section{REFERENCES}

[1] R. Brinkmann, Low Frequency Linear Colliders, Proceedings EPAC 94, London, England, 1994.

[2] R. Brinkmann, G. Materlik, J. Rossbach, A. Wagner (Eds.), Conceptual Design of a $500 \mathrm{GeV} \mathrm{e} \mathrm{e}^{+} \mathrm{e}^{-}$Linear Collider with Integrated X-Ray Laser Facility, DESY 1997-048 and ECFA 1997-182.

[3] Proposal for a TESLA Test Facility, DESY Report TESLA 93-01.

D. A. Edwards (Ed.), TESLA Test Facility Linac - Design Report, DESY Report TESLA 95-01.

[4] J. Rossbach (Ed.), A VUV FEL at the TESLA Test Facility Conceptual Design Report, DESY Rep. TESLA-FEL 95-03.

[5] T. Garvey et al., First Beam Test of the TTF Injector, Proceedings PAC 97, Vancouver, Canada, 1997.

[6] E. Colby et. al., Experimental Testing of the TTF RF Photoinjector, Proceedings PAC 97, Vancouver, Canada, 1997. S. Schreiber, Experiments with the RF gun based Injector for the TESLA Test Facility Linac, this conference.

[7] A. Gössel et al., Vertical and Horizontal Test Results in Comparison with Performance in the TTF Linac, $8^{\text {th }}$ Workshop on RF Superconductivity, Abano Terme, Italy, 1997.

[8] S. Wolff, The Infrastructure for the TESLA Test Facility, Proceedings PAC 95, Dallas, USA, 1995.

[9] C. Crawford et al., High Gradients in Linear Collider Superconducting Accelerator Cavities by High Pulsed Power to Suppress Field Emission, Part. Acc. 49, pp. 1-13, 1995.

[10] Q. S. Shu et al., An Advanced Rotating T-R Mapping \& its Diagnoses of the TESLA 9-cell Superconducting Cavities, Proceedings PAC 95, Dallas, USA, 1995.

[11] M. Pekeler, Test Results on the Superconducting 9-cell 1.3 GHz Cavities for the TESLA Test Facility Linac, Proceedings EPAC 98, Stockholm, Sweden, 1998.

[12] W. Singer et al., Diagnostics of Defects in High Pure Niobium, $8^{t h}$ Workshop on RF Superconductivity, Abano Terme, Italy, 1997.

[13] A. Gamp et al., Experience with the Control of the Vector Sum at the TESLA Test Facility, Proceedings EPAC 98, Stockholm, Sweden, 1998.

[14] E Kako et al., Improvement of Cavity Performance by Electropolishing in the $1.3 \mathrm{GHz} \mathrm{Nb}$ Superconducting Cavities, this conference.

[15] V. Palmieri, New Technologies in Superconducting Cavity Fabrication, Proceedings Applied Superconductivity Conference, Palm Desert, USA, 1998.

[16] H. Kaiser et. al, Hydroforming of Seamless Niobium Cavity of TESLA Shape, Proc. HEACC 98, Dubna, Russia, 1998.

[17] P. Kneisel and V. Palmieri, Development of Seamless Niobium Cavities for Accelerator Application, this conference.

[18] S. Bousson et al., A New Fabrication and Stiffening Method of SRF Cavities, Proc. EPAC 98, Stockholm, Sweden, 1998.

[19] J. Sekutowicz et al., Superconducting Superstructure for the TESLA Collider, Proceedings EPAC 98, Stockholm, Sweden, 1998 and DESY Report TESLA 98-08. 